\title{
A pipelined SAR ADC with gain-stage based on capacitive charge pump
}

\author{
Kairang Chen ${ }^{1} \cdot$ Atila Alvandpour ${ }^{1}$
}

Received: 18 May 2016/Revised: 30 September 2016/Accepted: 4 October 2016/Published online: 11 October 2016

(c) The Author(s) 2016. This article is published with open access at Springerlink.com

\begin{abstract}
This paper presents a 14-bit, tunable bandwidth two-stage pipelined successive approximation analog to digital converter which is suitable for low-power, cost-effective sensor readout circuits. To overcome the high DC gain requirement of operational transconductance amplifier in the gain-stage, the multi-stage capacitive charge pump (CCP) was utilized to achieve the gain-stage instead of using the switch capacitor integrator. The detailed design considerations are given in this work. Thereafter, the 14-bit ADC was designed and fabricated in a low-cost $0.35-\mu \mathrm{m}$ CMOS process. The prototype ADC achieves a peak SNDR of $75.6 \mathrm{~dB}$ at a sampling rate of $20 \mathrm{kS} / \mathrm{s}$ and $76.1 \mathrm{~dB}$ at $200 \mathrm{kS} / \mathrm{s}$ while consuming 7.68 and $96 \mu \mathrm{W}$, respectively. The corresponding FoM are 166.7 and $166.3 \mathrm{~dB}$. Since the bandwidth of CCP is tunable, the ADC maintains a SNDR $>75 \mathrm{~dB}$ upto $260 \mathrm{kHz}$. The core area occupied by the ADC is $0.589 \mathrm{~mm}^{2}$.
\end{abstract}

Keywords Capacitive charge pump - Two-stage pipelined SAR ADC · OTA · Switch capacitor integrator

\section{Introduction}

Wireless sensor networks are employed in many applications, such as monitoring bio-potential signals, environmental information and interactive multimedia. These

Kairang Chen

kairang.chen@liu.se

Atila Alvandpour

atila.alvandpour@liu.se

1 Department of Electrical Engineering, Linköping University, Linköping, Sweden applications require high-resolution ( $>12$ bits), low-speed (several kS/s) analog-to-digital converters (ADCs) [1]. Such sensor nodes are usually powered by batteries or energyharvesting sources $[2,3]$ hence low power consumption is primary for the constituent ADCs. Normally, tens or hundreds of autonomously powered sensor nodes are utilized to capture and transmit data to the central processor. Hence it is profitable to fabricate the relevant electronics, such as the ADCs, in a low-cost standard CMOS process.

The successive approximation register analog-to-digital converters (SAR ADCs) can provide highly power efficiency solution at moderate resolutions, but achieving a SAR ADC with effective number of bits (ENOB) beyond 12 bits shows enormous challenges due to the influence from comparator noise and capacitor mis-matching [4-6]. The SAR-assisted pipeline ADC is an energy-efficient architecture for high resolution [7]. Such architecture consists of two independent sub-SAR ADCs coupled by a gain-stage. The need of a high-accuracy comparator can be obviated by incorporating a SAR ADC as the sub-ADC in the pipeline stage. However, an additional gain-stage is inevitable to amplify the residue signal. Normally, the switch capacitor (SC) integrator is the primary choice to realize the gain-stage, but a high-gain operational transconductance amplifier (OTA) is necessary to amplify the residue with sufficient accuracy. Since the open-loop DC gain requirement of OTA grows exponentially with the total resolution of the ADC, the design of high-gain OTA is a major challenge for implementing high-resolution pipelined SAR ADC. Meanwhile, as shown in [7-9], the high gain OTA is always a power-hungry block. So, a lowerpower alternative way, such as the dynamic amplifier $[10,11]$, which allows to switch off the OTA during the reset phase but still needs a high open-loop DC gain. In [12], an open-loop amplifier as the gain stage was offered. 
Although the open-loop solution shows a low DC gain, this voltage gain will be deeply influenced by the process variation. So the extra calibration technique is inevitable.

To overcome the high DC gain requirement, in this paper, we employ the design, analysis and implementation of a 14-bit, tunable bandwidth two-stage pipelined SAR ADC in $0.35-\mu \mathrm{m}$ CMOS process which uses a three-stage capacitive charge pump (CCP) as the gain-stage instead of using SC integrator. Since the basic CCP cell can provide a gain of 2 [13], m-stage CCP can be connected in series to achieve a gain of $2^{m}$. Combining the gain reduction solution as shown in [14], three-stage CCP was utilized to achieve a stage gain of 8 . Since the gain reduction solution requires increased sampling capacitance for the second stage sub-ADC to compensate for the reduced signal swing [8], the attenuation DAC [15] was chosen to alleviate the increased capacitance requirement in the second stage. The segmented capacitive array DAC [16] was implemented in the first stage which meets the targeted static linearity with a lower unit capacitance. The prototype ADC achieves a peak SNDR of $75.6 \mathrm{~dB}$ at a sampling rate of $20 \mathrm{kS} / \mathrm{s}$ and $76.1 \mathrm{~dB}$ at a sampling rate of $200 \mathrm{kS} / \mathrm{s}$ while consuming 7.68 and $96 \mu \mathrm{W}$, respectively.

This paper is organized as follows. Section 2 introduces the proposed pipelined SAR ADC architecture and the operating sequence. Section 3 describes the details of circuit implementation. The measurement results are presented in Sect. 4, followed by the conclusions in Sect. 5.

\section{Architecture of the proposed two-stage pipelined SAR ADC}

Figure 1 shows the proposed two-stage pipelined SAR ADC architecture. It consists of $N_{1}$ bits SAR ADC with segmented binary-weighted capacitive DACs, a multi-stage
CCP and a $N_{2}$ bits SAR ADC with split binary-weighted capacitive DACs. For the pipelined SAR ADC, although the resolution of the first stage is $N_{1}$, the accuracy for the first stage must meet the total resolution $N$ and also the matching constraints necessitate large unit capacitor in the capacitive array which entails large power consumption and chip area. For the segmented DAC with a segmentation degree $k$, the unit capacitor value is $2^{k-1}$ times lower than the unit capacitor for the conventional binary-weighted DAC in order to meet the same targeted static linearity [17]. Hence the SAR ADC with segmented binary-weighted capacitive DAC serves as the first stage in order to relax the unit capacitor value and the active chip area. The multistage CCP works as the gain-stage to amplify the residue signal with a inter-stage gain of $2^{m}$ where the $m=N_{1}-r$ and $r$ is the gain reduction factor [14]. Since the basic CCP cell applies an ideal gain of 2 [13], $N_{1}-r$ stages are necessary to achieve a gain of $2^{N_{1}-r}$. The inter-stage gain is reduced by $2^{r}$ times, which means an extra capacitor $C_{x}=$ $\left(2^{r-1}-1\right) C_{t o t 2}$ is required for the second stage DAC [14] to compensate for the reduced signal swing where the $C_{t o t 2}$ represents the total capacitance of second DAC. So the SAR ADC with split binary-weight capacitive DAC is chosen to alleviate the increased capacitance requirement in the second stage.

Figure 2 gives the timing diagram of the proposed architecture with three-stage CCP. The input signal $V_{\text {in }}$ is sampled through the switch $\mathrm{S}_{1}$ at the phase $f_{s 1}$ marks the beginning of the conversion. The residue signal is generated after $N_{1}+1$ steps conversions. Thereafter, the residue signal is sampled and amplified during phase $\phi_{1 \_s}$ and $\phi_{1 \_a}$ respectively. The output signal from the first CCP cell will be sampled and amplified by the following stage at phase $\phi_{2 \_s}$ and $\phi_{2 \_}$. So the rest can be done in the same manner until the residue signal is magnified by 8 times.



Fig. 1 Proposed two-stage pipelined SAR ADC architecture 


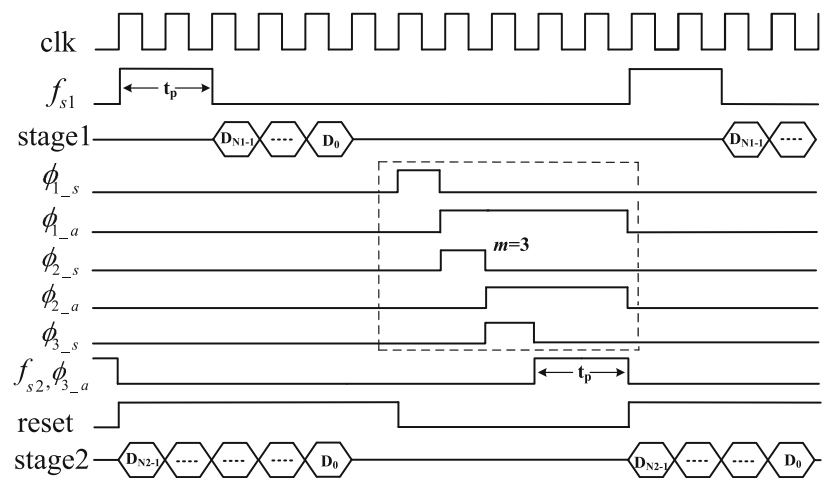

Fig. 2 Timing diagram for proposed pipelined SAR ADC architecture with $m=3$

The final output also as the input signal of the second stage will be involved in the following ADC conversion at phase $f_{s 2}\left(\phi_{3 \_a}\right)$. From Fig. 2, the clock frequency can be derived as

$f_{c l k}=\left(N_{1}+2 t_{p}+m+1\right) f_{s}$,

where $m=N_{1}-r$ is the stage number of gain-stage and $t_{p}$ is the duration of sampling frequency which is set to $2 / f_{c l k}$.

\section{Proposed pipelined SAR ADC architecture implementation}

\subsection{Multi-stage CCP analysis and implementation}

The multi-stage CCP architecture is shown in Fig. 1, consisting of several basic charge pump cell [13]. For each cell, it supplies a gain of 2 . Thus, the multi-stage CCP could achieve a gain of $2^{m}$ ideally. In this architecture, the only active circuitry is the unity gain buffer with a capacitive load of $C_{m}$. Proper choice of the $C_{m}$ is crucial for keeping the noise level below the corresponding quantization noise. Meanwhile the choice of the stage number $m$ is also elaborated upon in this section.

\subsubsection{Noise analysis}

The thermal noise, introduced by the on-resistance of the sampling switch, is the main noise source for the first stage. This noise is sampled by $C_{1}$ during $\phi_{1 \_s}$ which is given by

$\overline{v_{n, \phi_{1}-s}^{2}}=\frac{k T}{C_{1}}$,

where $k$ is the Boltzmann constant and $T$ is the absolute temperature.

For the second stage, the noise is determined by three noise sources which are the noise from the switch in the stage itself, the noise from the previous sampling switch

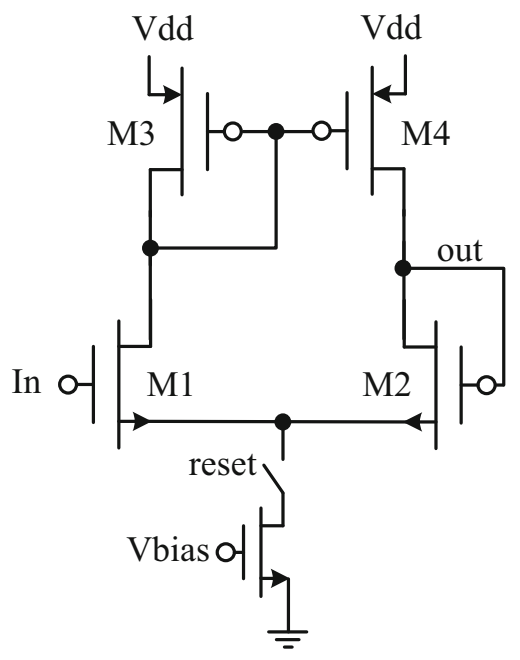

Fig. 3 OTA in unity gain configuration

and also the noise from unity gain buffer respectively. The OTA in unity gain configuration as shown in Fig. 3 is employed in this work.

Because the unit gain frequency of the buffer is $\frac{g_{m 1}}{C_{2}}$, where the $g_{m 1}$ is the transconductance of M1 in Fig. 3, the total noise of the resistor is shaped by the low-pass characteristic. So, the total noise power of the resistors is given by

$\overline{v_{n \_R_{o n}, \phi_{2} \_s}^{2}}=\frac{16 k T R_{o n}}{4 \tau}$.

By using $\tau=\frac{C_{2}}{g_{m 1}}$, where $\tau$ is the time constant, the total noise power from resistor is

$\overline{v_{n \_}^{2} R_{o n}, \phi_{2} \_s}=\frac{k T}{C_{2}} 4 g_{m 1} R_{o n}$.

For the unity gain buffer, the ouput noise power is

$\overline{v_{n \_ \text {OTA }, \phi_{1} \_a}^{2}}=\frac{4 k T}{3 C_{2}}\left(1+\frac{g_{m 3}}{g_{m 1}}\right)$,

where $g_{m 3}$ is the transconductance of M3 in Fig. 3. So, the total noise power sampled by $C_{2}$ at phase $\phi_{2}{ }_{s}$ is given by the sum of Eqs. (4) and (5) which is

$\overline{v_{n \_t o t, \phi_{2} \_s}^{2}}=\frac{k T}{C_{2}} 4 g_{m 1} R_{\text {on }}+\frac{4 k T}{3 C_{2}}\left(1+\frac{g_{m 3}}{g_{m 1}}\right)$,

with $g_{m 1}=27.2 \mu \mathrm{A} / \mathrm{V}, g_{m 3}=10.9 \mu \mathrm{A} / \mathrm{V}, R_{o n}=8.8 \mathrm{k} \Omega$ and $C_{2}=2 \mathrm{pF}$, the total noise power $\overline{v_{n \_t o t, \phi_{2} s}^{2}}=5.85 \times 10^{-9}$ $V^{2}$ at phase $\phi_{2 s_{s} s}$. To verify the noise estimation, the test bench with the first stage was built and simulated in 0.35$\mu \mathrm{m}$ CMOS process.

Fig. 4 shows the simulation result that is very close to the calculation. Hence the total input referred noise for the first cell is: 
$\overline{v_{n}^{2}{ }_{-t o t, i n \_c}}=\frac{k T}{C_{1}}+\frac{1}{\left(G_{s}\right)^{2}}\left(\frac{k T}{C_{2}} 4 g_{m 1} R_{\text {on }}+\frac{4 k T}{3 C_{2}}\left(1+\frac{g_{m 3}}{g_{m 1}}\right)\right)$,

where $G_{s}$ is the stage gain which is 2 ideally for single stage.

The above noise analysis just focus on one single stage. If this single stage is followed by another stage, the noise of the following stage will contribute to the total noise of the previous stage. Equation (6) could stand for the total output noise for each cell. So for the multi-stage charge pump, the total input referred noise can be derived as

$\overline{v_{n \_t o t, i n p u t}^{2}}=\frac{k T}{C_{1}}+\frac{\overline{v_{n \_t o t, \phi_{2}-s}^{2}}}{\left(G_{s}\right)^{2}}+\frac{\overline{v_{n \_t o t, \phi_{3}-s}^{2}}}{\left(G_{s}\right)^{4}}+\ldots+\frac{\overline{v_{n \_t o t, \phi_{m \_s}}^{2}}}{\left(G_{s}\right)^{2^{m-1}}}$.

It can be readily seen from (8) that more stages will contribute more noise to the total input referred noise. Thus, a large $C_{1}$ is inevitable. For a three-stage CCP, in order to maintain the input referred noise is below the quantization noise of a 14-bit ADC. From (8) and also reusing the parameters from (6), the minimum $C_{1}=2.7 \mathrm{pF}$ under the assumption that the $C_{2}=C_{3}=2 \mathrm{pF}$. Meanwhile more stages also indicate more power consumption. Hence, proper choice of stage number is important. Since the gain bandwidth of unity-gain buffer can be changed by tuning the $V_{\text {bias }}$ in Fig. 3, the bandwidth of CCP is tunable.

\subsubsection{Gain requirement and power analysis}

The voltage gain of SC integrator can be shown as

$G=\frac{1}{\beta}\left(1-\frac{1}{\beta A}\right)$,

where $\beta=2^{r-N_{1}}$ is the feedback factor with the gain reduction factor $r$ and $\Delta e=\frac{1}{\beta A}=\frac{1}{2^{N-N_{1}}}$ is the gain error caused by the finite open-loop DC gain of the OTA [14]. In order to obtain the same voltage gain by using the CCP architecture, $m$ charge pump stages should be connected in series. The gain of one stage is

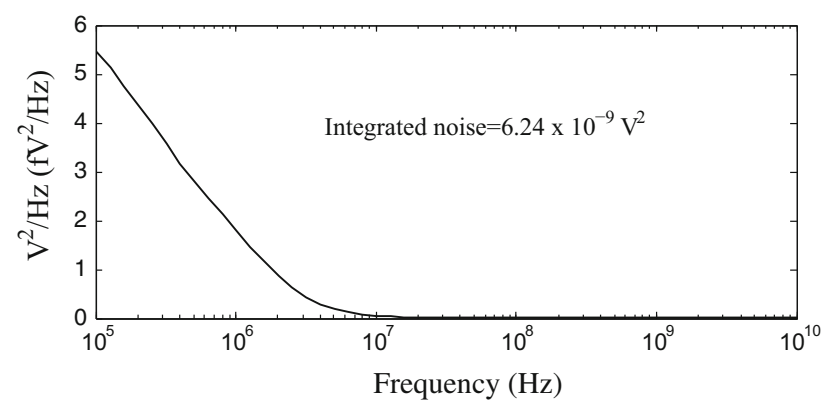

Fig. 4 The total noise in $C_{2}$ at phase $\phi_{2 \_} s$
$G_{s}=2 G_{b u f}$,

thus

$\left(2 G_{b u f}\right)^{m}=\frac{1}{\beta}(1-\Delta e)$,

where the $G_{b u f}$ is unity gain of buffer which can be expressed as

$G_{b u f}=1-\frac{1}{A_{b u f}}$.

Substituting (12) into (11) and replacing $\beta, \Delta e$ with $2^{r-N_{1}}$ and $2^{N_{1}-N}$ respectively. The DC gain $A_{b u f}$ for the OTA in unity gain configuration can be formulated as

$A_{b u f}=\frac{1}{1-\sqrt[N_{1}-r]{\left(1-\frac{1}{2^{N-N_{1}}}\right)}}$.

By using $A=2^{N-r}[14]$ and (13), Fig. 5 plots the DC gain requirement of OTA in SC and CCP for a 14-bit ADC with $N_{1}=7$. It is clear to note that the DC gain for SC grows exponentially whereas it increases linearly for CCP's DC gain. For instance, to achieve a voltage gain with $\Delta e=\frac{1}{2^{7}}$, the open-loop DC gain of OTA in unity gain configuration is $A_{b u f}=383(51.7 \mathrm{~dB})$. However, this DC gain value for OTA in SC integrator is $1024(60.2 \mathrm{~dB})$. Although the DC gain is reduced, $\mathrm{m}$-stage CCP is needed to achieve a voltage of $2^{m}$. Hence it is worth to analyze the power consumption of CCP.

Following the analysis in [18], the power consumption for a single-stage transconductance amplifier with capacitive feedback is

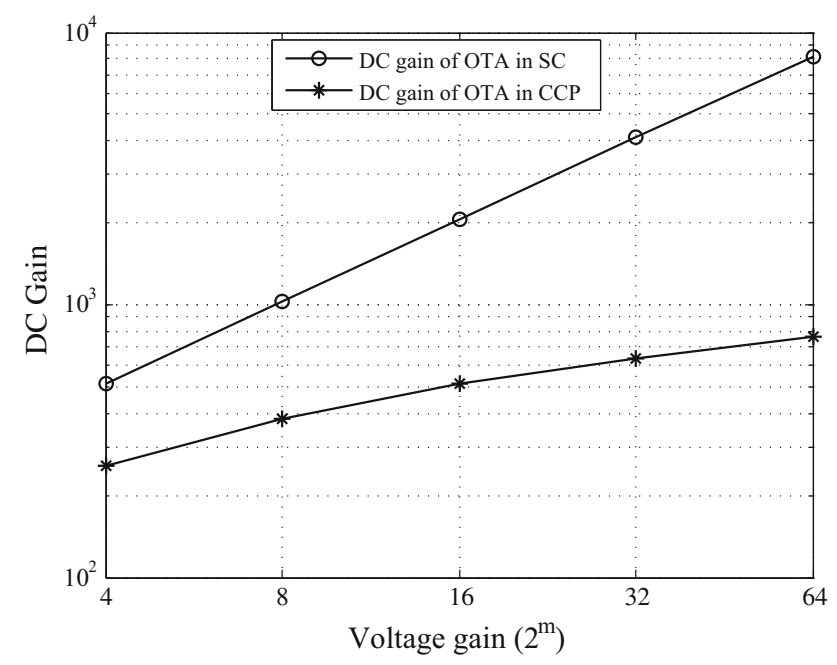

Fig. 5 DC gain requirement of OTA in $\mathrm{CCP}$ and $\mathrm{SC}$ architecture versus voltage gain $\left(2^{m}\right)$ 
$P_{\text {OTA_G }}=2 V_{F S}^{2} f_{S} C_{L A}\left(1+(1+|G|) N \ln 2 \cdot \frac{V_{\text {eff }}}{V_{F S}}\right)$,

where $C_{L A}$ is the capacitive load of OTA, $N$ is total resolution of $\mathrm{ADC}, V_{F S}$ is the full-scale range of the ADC and $G$ is the voltage gain. For a MOS transistor in strong inversion, the parameter $V_{\text {eff }}=\left(V_{g s}-V_{T}\right) / 2$, where $V_{g s}$ and $V_{T}$ are the gate-to-source and threshold voltages respectively [18]. By replacing $G$ with (9), the (14) can be expressed as

$$
P_{\text {OTA } \_} G=2 V_{F S}^{2} f_{s} C_{L A}\left(1+\left(1+\left|2^{m}\left(1-\frac{2^{m}}{A}\right)\right|\right) \cdot N \ln 2 \cdot \frac{V_{\text {eff }}}{V_{F S}}\right) .
$$

For the $\mathrm{CCP}$, the only active circuitry is the unity gain buffer which dominates the total power consumption of CCP. For this OTA, the power consumption is

$P_{\text {OTA_1 }}=2 V_{F S}^{2} f_{s} C_{L A}\left(1+\left(1+\left|1-\frac{1}{A_{\text {buf }}}\right|\right) N \ln 2 \cdot \frac{V_{\text {eff }}}{V_{F S}}\right)$.

To achieve a gain of $2^{m}, m$ unity gain buffers are needed. To simplify the analysis, we assume that the power consumption of each buffer is equal to the first one's and also ignore the power consumption from switches. Normally, the implementation is fully differential. Hence the total power for $m$-stage CCP is

$P_{\text {OTA } \_m}=2 m \cdot P_{\text {OTA } \_1}$.

With $V_{F S}=3.3 \mathrm{~V}, V_{\text {eff }}=0.3 \mathrm{~V}$, Fig. 6 plots the OTA energy consumption in $\mathrm{CCP}$ and $\mathrm{SC}$ architecture according to (15) and (17) as a function of $m$. To find the influence from larger $m, N=14$ and $N=16$ was chosen respectively but with the same load capacitance of $3 \mathrm{pF}$. In practice, the load capacitance increases along with the growth of resolution. From Fig. 6, the OTA power in SC grows exponentially but linearly in CCP. The CCP consumes more power than $\mathrm{SC}$ with lower voltage gain due to more unity gain buffers are used. However, the CCP is more power efficient than SC with high voltage gain which shows a power saving potential for designing higher resolution ADC.

To verify the analysis, the gain-stage utilized the CCP and SC integrator were simulated in $0.35-\mu \mathrm{m}$ CMOS. In order to find the impacts from the stage number, the voltage gain of 8 and 64 were chosen for the simulation. To achieve the high gain requirement of the OTA in SC integrator, the two-stage OTA with Miller compensation was utilized. During the simulation, half of the sampling period was used as the residue amplification phase. The simulation results show that the DC gain requirement in $\mathrm{CCP}$ architecture is $51.8 \mathrm{~dB}$ to obtain a voltage gain of 7.94

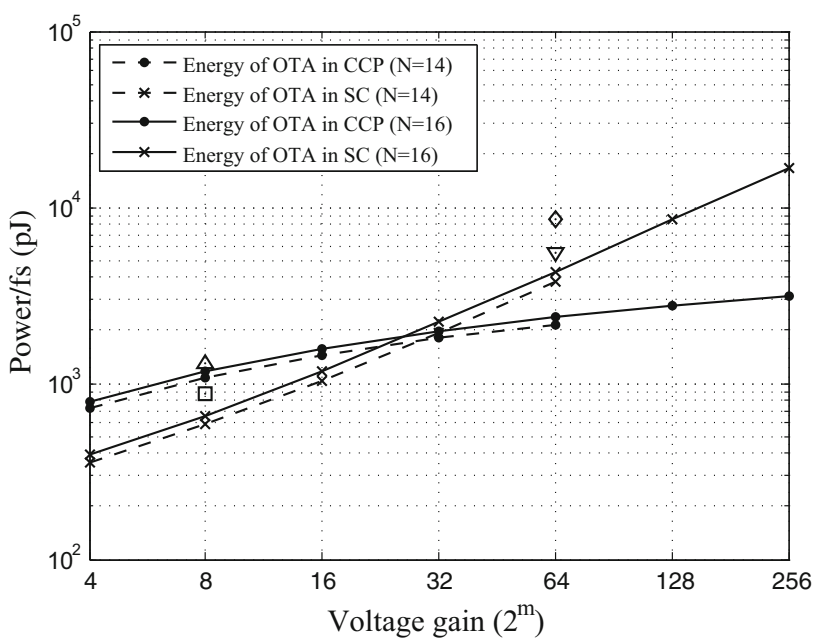

Fig. 6 OTA energy consumption in CCP and SC architecture versus voltage gain $\left(2^{m}\right)$ with $N=14, N_{1}=7$ and $N=16, N_{1}=9$ together with the simulation results (triangle: $\mathrm{CCP}$; square: $\mathrm{SC}$; inverted triangle: $\mathrm{CCP}$; diamond: $\mathrm{SC}$ )

and the corresponding power is $1.17 \mu \mathrm{W}$ at $1 \mathrm{kS} / \mathrm{s}$ with $C_{1}=3 \mathrm{pF}, C_{2}=C_{3}=2 \mathrm{pF}$. For the $\mathrm{SC}$ integrator, the power is $0.87 \mu \mathrm{W}$ at $1 \mathrm{kS} / \mathrm{s}$ with $C_{L A}=3 \mathrm{pF}$ but the DC gain is $61.7 \mathrm{~dB}$ to achieve the same voltage gain. The CCP's power is higher than SC's due to more unity gain buffers were used. When it comes to the voltage gain of 64 , the power of $\mathrm{CCP}$ is $5.53 \mu \mathrm{W}$ at $1 \mathrm{kS} / \mathrm{s}$ with $C_{1}=$ $3 \mathrm{pF}, C_{2}=C_{3}=C_{4}=C_{5}=C_{6}=2 \mathrm{pF}$ whereas the power of SC is $8.67 \mu \mathrm{W}$ with $C_{L A}=3 \mathrm{pF}$ at the same sampling rate and the corresponding DC gain requirements are $58 \mathrm{~dB}$ for unity gain buffer and $78.5 \mathrm{~dB}$ for OTA in SC to get a voltage gain of 63.4. The four simulation results were added into Fig. 6 as well and the above relevant capacitors values are obtained from (8). In Fig. 6, the simulated results are somewhat higher than the predicted results. This is due to the design margin taken into the OTA design.

In this work, the solution of $m=3$ has been applied to our 14-bit two-stage pipelined SAR ADC project. It should be pointed out that the main purpose of this work was to verify the overall functionality of the proposed multi-stage capacitive charge pump. Compared to the conventional SC integrator, the CCP gain-stage with a voltage gain of 8 is not power-efficient choice for design of a 14-bit ADC. It remains as a future work to find a low-power analog buffer instead of using the unity-gain OTA. However, Fig. 6 suggests that the presented gain-stage would result in higher power efficiency for higher resolutions, for example for 16-bit ADC with 9-bit in the first-stage.

The implementation of unity-gain buffer is shown in Fig. 3 which has a DC gain of $51.8 \mathrm{~dB}$. To determine the impact of process variations and device mismatch on the unity-gain, 200 Monte Carlo simulations were performed. With an external $V_{\text {bias }}$ of $0.48 \mathrm{~V}$, Table 1 summarizes the 
simulated unity-gain of OTA across process corners, device mismatch and at a temperature range of -40 to $85{ }^{\circ} \mathrm{C}$.

As the table shows the unity-gain OTA shown in Fig. 3 maintains its gain sufficiently constant. To meet the noise requirement of 14-bit $\mathrm{ADC}$, the $C_{1}=3 \mathrm{pF}, C_{2}=C_{3}=2$ $\mathrm{pF}$ were chosen. Since the input of gain-stage is discrete time, the normal NMOS transistor was used to achieve the switch. It should be mentioned that one more unity gain buffer is needed between the first stage ADC and the input of gain-stage. The gain error caused by this buffer will be compensated by tuning the reference voltage of second ADC.

\subsection{First-stage SAR ADC implementation}

For the 14-bit two-stage pipelined SAR ADC, the $N_{1}=7$ was allocated to the first-stage. Aiming to reduce the power consumption and active area of the first-stage, The segmented binary-weighted capacitive DAC was implemented in the first SAR ADC. The reasons are as follows. Since the static linearity of the first-stage is dominated by the capacitor mismatch in the corresponding DAC. Following the detailed analysis in [14], the mismatch-limited unit capacitor for the first-stage with conventional binaryweighted capacitive DAC is

$C_{u 1}>4.5 K_{\sigma}^{2} K_{c} 2^{2\left(N-N_{1}\right)}\left(2^{N_{1}}-1\right)$,

and for the segmented binary-weighted capacitive DAC, the mismatch-limited unit capacitor is

$$
C_{u 1}>4.5 K_{\sigma}^{2} K_{c} 2^{2\left(N-N_{1}\right)}\left(2^{N_{1}-k+1}-1\right),
$$

where $K_{\sigma}$ is the mismatch parameter, $K_{c}$ is the capacitor density and $k$ is the segmented degree. Comparing (18) and (19), it is seen that the $C_{u 1}$ for the segmented DAC is $2^{k-1}$ times lower than that of the conventional DAC. For the poly-insulator-poly (PIP) capacitor in $0.35-\mu \mathrm{m}$ CMOS process, $K_{\sigma}=0.45 \% \mu \mathrm{m}$ and $K_{c}=0.86 \mathrm{fF} / \mu m^{2}$. From (18), the minimum mismatch-limited unit PIP capacitor is $163 \mathrm{fF}$. However, the segmented DAC requires only $C_{u 1}=$ $40.8 \mathrm{fF}$ with a segmented degree $k=3$. Therefore, it will avail power and active area savings by using a segmented

Table 1 Unity-gain OTA performance

\begin{tabular}{llll}
\hline$V_{d d}=3.3 \mathrm{~V}$ & \multicolumn{2}{l}{ Unity-gain } \\
\cline { 2 - 4 } Temperature $\left({ }^{\circ} \mathrm{C}\right)$ & Min. & Max. & $3 \sigma$ \\
\hline-40 & 0.9963 & 0.9978 & $740.7 \mathrm{e}-6$ \\
0 & 0.9968 & 0.9978 & $563.7 \mathrm{e}-6$ \\
27 & 0.9968 & 0.9977 & $542.1 \mathrm{e}-6$ \\
85 & 0.9967 & 0.9977 & $655.8 \mathrm{e}-6$ \\
\hline
\end{tabular}

capacitive DAC in the first stage. Here, the minimum mismatch-limited unit capacitance $(40.8 \mathrm{fF})$ is calculated under the assumption that the sampling switch is ideal. In practice, the sampling switch introduces the charge injection and clock feed-through errors which cause additional harmonic distortions. Hence a large unit capacitor of 117.7 $\mathrm{fF}$ was required by simulations to achieve the targeted 14-bit ADC performance.

It should be noted that the segmented DAC requires a binary-to-thermometer decoder which lead to extra power consumption and chip area. Larger segmentation degree indicates that the circuit complexity, area occupation and power increase. In [14], the optimal segmentation degree range is $3 \leq k \leq 5$. In this work, a segmentation degree of $k=3$ was chosen for the first stage DAC as a trade-off among lower unit capacitor value, increased circuit complexity and power consumption in the digital logic. Figure 7 shows the schematic of the 3-to-7 binary-tothermometer decoder required for the unary-weighted 3-bit DAC segment which consists of basic logic gates.

Figure 8 shows the control logic circuits which were used to generate the time sequence as shown in Fig. 2. The block diagram of the SAR control logic for the first-stage consisting of two separate shift registers is shown in Fig. 8(a). The upper DFFs chain generate the bit approximation pulses while the DFFs in lower row store the output $C_{\text {out }}$ of the comparator. Finally, the switch control signals (D13...D7) for the DAC are generated which will be involved in the ADC conversion. The control logic of the three-stage CCP is illustrated in Fig. 8(b). The first four DFFs are connected in series condition which are used to generate the sampling phase $\left(\phi_{1 \_s} \ldots \phi_{3 \_a}\right)$. By choosing the $\phi_{2 \_s}, \phi_{3 \_s}$ and $\phi_{3 \_a}$ as the input of an OR gate, the amplifying phase $\phi_{1 \_a}$ can be obtained from the output of OR gate. The $\phi_{2 \_a}$ can be implemented through the same way. An AND gate was utilized to create the reset signal by connecting the reverse of four sampling phase. The additional two DFFs with an OR gate are triggered by a "set" signal which subsequently create the sampling signal $f_{s 1}$ for

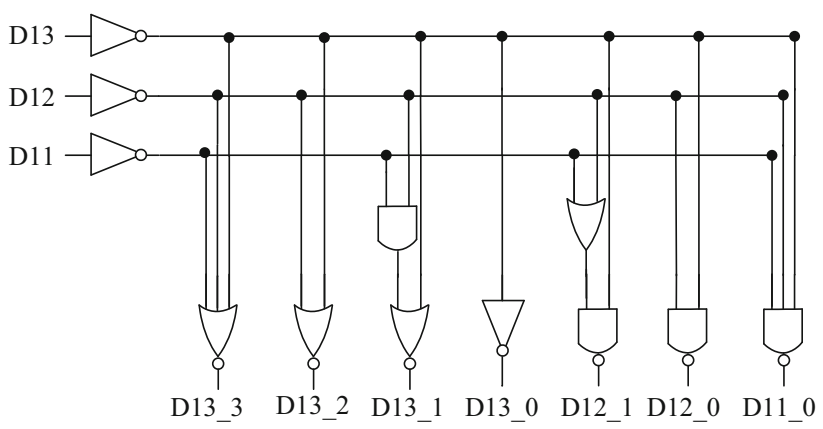

Fig. 7 3-to-7 binary to unary thermometer decoder 
(a)

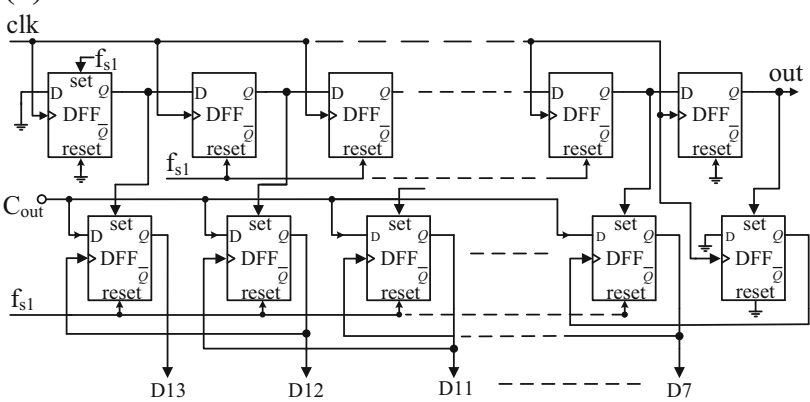

(b)

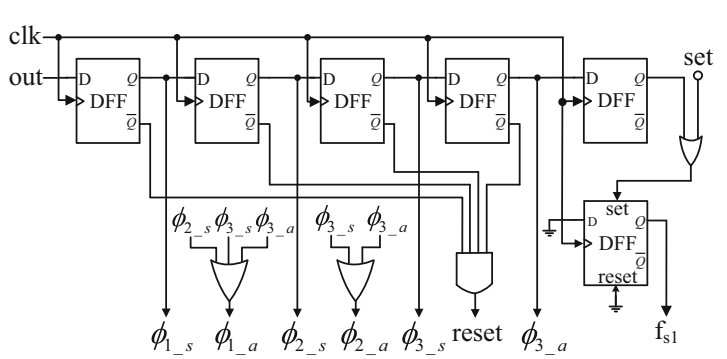

Fig. 8 Control logic. a SAR control logic of first stage. b Control logic of three-stage CCP

the first-stage and also the $f_{s 1}$ as the start signal is connected to the relevant position as shown in Fig. 8(a).

For the input sampling switch $S_{1}$ in Fig. 1, the conventional bootstrapped switch [19] is used for improved linearity. Simulation results indicate a linearity corresponding to 17.6-bit for $\mathrm{S}_{1}$ with a sampling capacitance of $20 \mathrm{pF}$ and $20 \mathrm{kS} / \mathrm{s}$ sampling frequency which is sufficient for the 14-bit resolution. The conventional dynamic latch comparator as shown in Fig. 9 is used to generate the digital output bits. Simple inverters have been used to implement the DAC switches.

\subsection{Second-stage SAR ADC implementation}

A 8-bit SAR ADC with attenuation capacitor based DAC forms the second-stage sub-ADC. The DAC consists of a 4-bit main-DAC and 4-bit sub-DAC. Because of $m=3$, the extra capacitor $C_{x}=112 C_{u 2}$ was added into the DAC. As the total capacitance of the second sub-ADC $\left(C_{t o t 2}\right)$ is also the load capacitance of the gain-stage, an unit PIP capacitor $C_{u 2}$ was chosen as $15.8 \mathrm{fF}$ yielding a $C_{\text {tot } 2}=2.0 \mathrm{pF}$ to maintain the total input-referred noise of the gain-stage [Eq. (8)] below the quantization noise of 14-bit ADC. Such choice also satisfies the thermal noise and mismatch requirements of the second-stage ADC's accuracy (11-bit). The SAR logic for the second-stage is similar to that shown in Fig. 8(a) except the reset signal should be connected to $\phi_{3 \_a}$ generated by control logic as shown in Fig. 8(b). Since the accuracy of this stage is 11-bit, a transmission gate was used to design the sampling switch $S_{2}$ as shown in

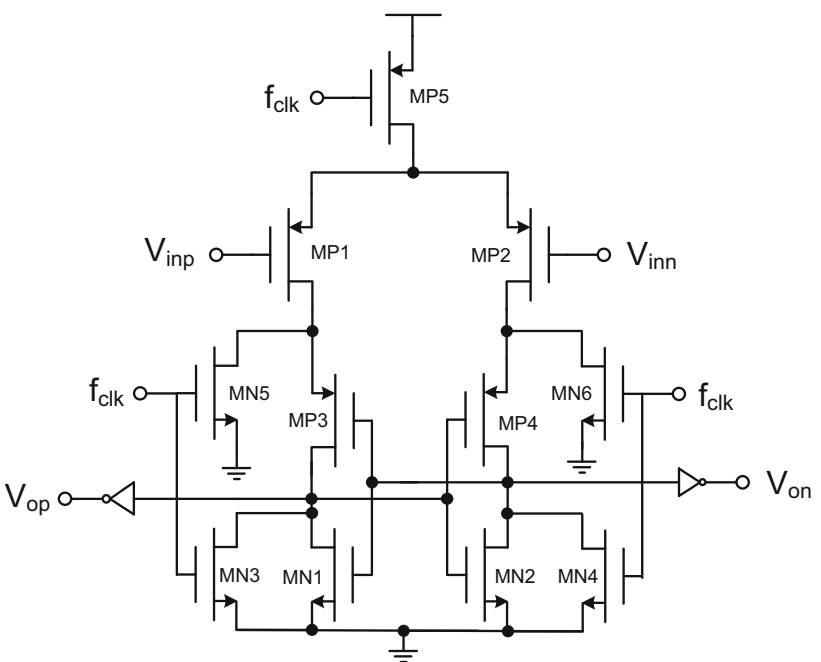

Fig. 9 Dynamic latch comparator

Fig. 1. The same dynamic latch comparator topology as in the first stage is used to generate the digital output bits.

\section{Measurement results}

The fully differential 14-bit ADC with a core area of $860 \mu \mathrm{m} \times 685 \mu \mathrm{m}$ was fabricated in a one-poly-four-metal (1P4M) $0.35-\mu \mathrm{m}$ CMOS process. The core was packaged in a JLCC44 package. Figure 10 shows the chip micrograph. The unmarked area in Fig.10 includes decoupling capacitors and I/O buffers.

At $V_{\text {bias }}=0.48 \mathrm{~V}$ as shown in Fig. 3, the measured FFT spectrum of the ADC operating at a sampling rate of $20 \mathrm{kS} /$ $\mathrm{s}$ with near-DC $(0.5127 \mathrm{kHz})$ and near-Nyquist $(9.526 \mathrm{kHz})$ input tones was shown in Fig. 11. The amplitude of the input signal was set to $-0.052 \mathrm{dBFS}$. A clock frequency of $\mathrm{f}_{c l k}=300 \mathrm{kHz}$ was used. The SNDR, SFDR and ENOB are 75.6, $90.9 \mathrm{~dB}$ and 12.27-bit respectively with the near-DC input tone. For the near-Nyquist input tone SNDR, SFDR and ENOB are 74.63, $90.79 \mathrm{~dB}$ and 12.1-bit respectively.

By increasing the $V_{\text {bias }}$ to $0.63 \mathrm{~V}$, the ADC can work at a sampling frequency of $200 \mathrm{kHz}$. Fig. 12 shows the measured FFT spectrum with near-DC and near-Nyquist input tones but under a sampling rate of $200 \mathrm{kS} / \mathrm{s}$. With the same amplitude of the input signal, the measured SNDR is 76.11 $\mathrm{dB}$, providing a 12.35-bit ENOB with near-DC input. For the near-Nyquist input, the ADC also achieves a $74.47 \mathrm{~dB}$ SNDR and 12.08-bit ENOB. The SNDR and SFDR over the range of input signal frequencies upto the Nyquist bandwidth for $20,200 \mathrm{kS} / \mathrm{s}$ are shown in Fig. 13. The SNDR $>74 \mathrm{~dB}$ is maintained upto the Nyquist bandwidth for the two sampling rates. 


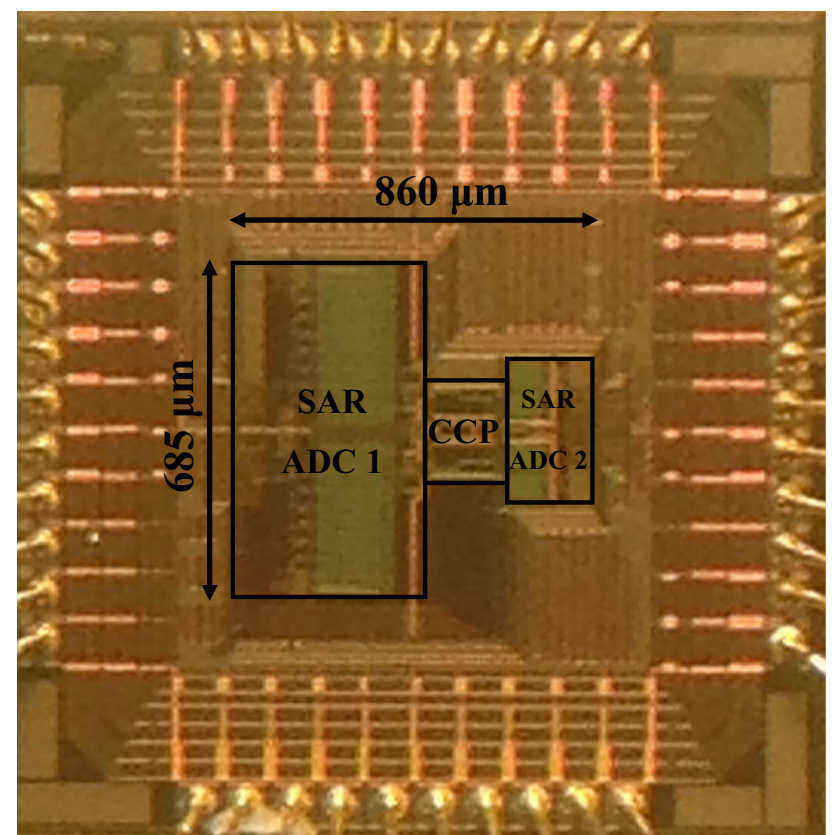

Fig. 10 Die micrograph
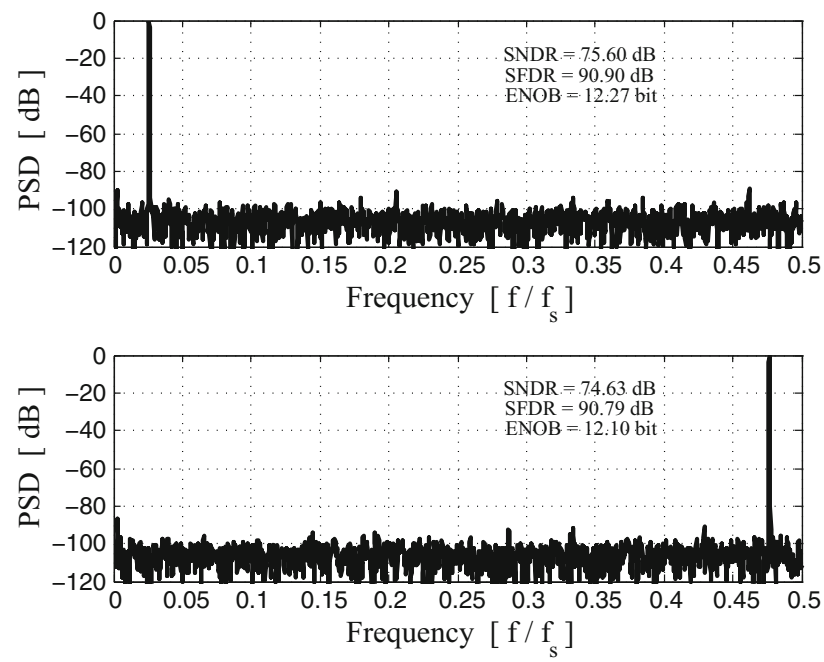

Fig. 11 Measured 4096-point FFT spectrums with near-DC $\left(f_{\text {in }}=\frac{105}{4096} f_{s}\right)$ and near-Nyquist $\left(f_{\text {in }}=\frac{1951}{4096} f_{s}\right)$ inputs at $20 \mathrm{kS} / \mathrm{s}$

Since the bandwidth of CCP is tunable, Fig. 14 shows the measured SNDR versus the sampling rate under the different $V_{\text {bias }}$ setting. By increasing the bandwidth of CCP, the proposed ADC achieves a SNDR $>75 \mathrm{~dB}$ upto $260 \mathrm{kS} /$ $\mathrm{s}$. Due to the speed limitation of comparator and sampling switch, the ADC performance drops fast after $f_{s}=260 \mathrm{kHz}$.

Histogram test was used to measure the static linearity of the ADC. A full swing, differential sinusoidal input tones $\left(f_{\text {in }}=14.64 \mathrm{~Hz}\right.$ at $f_{s}=20 \mathrm{kHz}, \mathrm{f}_{\text {in }}=146.48 \mathrm{~Hz}$ at $f_{s}=200 \mathrm{kHz}$ ) were applied to the ADC. The measured differential nonlinearity (DNL) and integral nonlinearity (INL) for 20 and $200 \mathrm{kS} / \mathrm{s}$ are shown in Figs. 15 and 16.
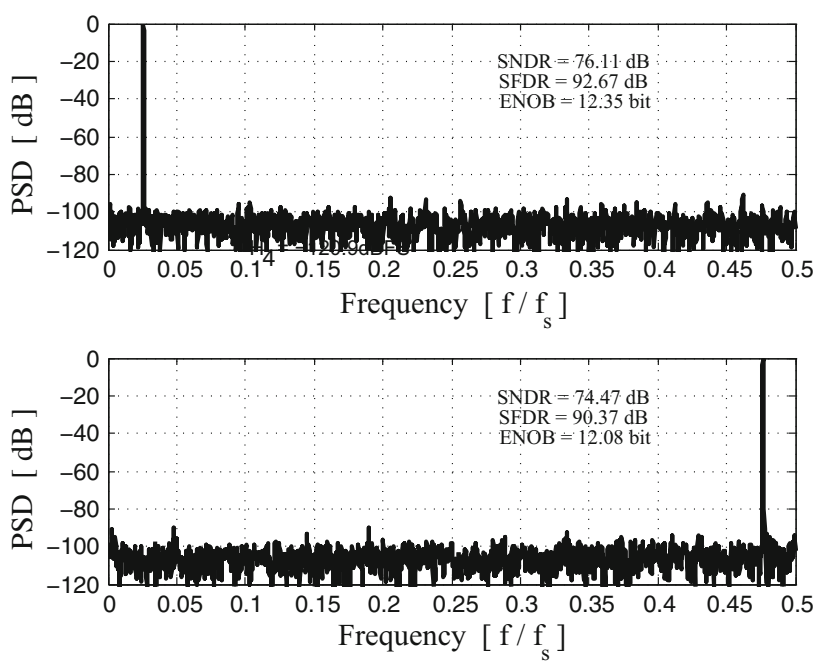

Fig. 12 Measured 4096-point FFT spectrums with near-DC $\left(f_{\text {in }}=\frac{105}{4096} f_{s}\right)$ and near-Nyquist $\left(f_{\text {in }}=\frac{1951}{4096} f_{s}\right)$ inputs at $200 \mathrm{kS} / \mathrm{s}$

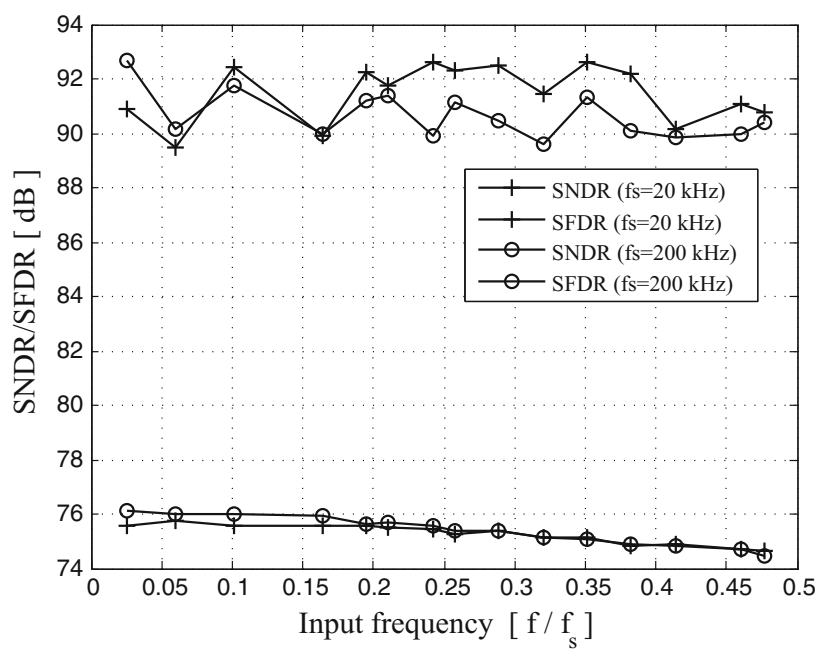

Fig. 13 Measured SNDR and SFDR at $20 \mathrm{kS} / \mathrm{s}\left(V_{\text {bias }}=0.48 \mathrm{~V}\right), 200$ $\mathrm{kS} / \mathrm{s}\left(V_{\text {bias }}=0.63 \mathrm{~V}\right)$ versus input frequency

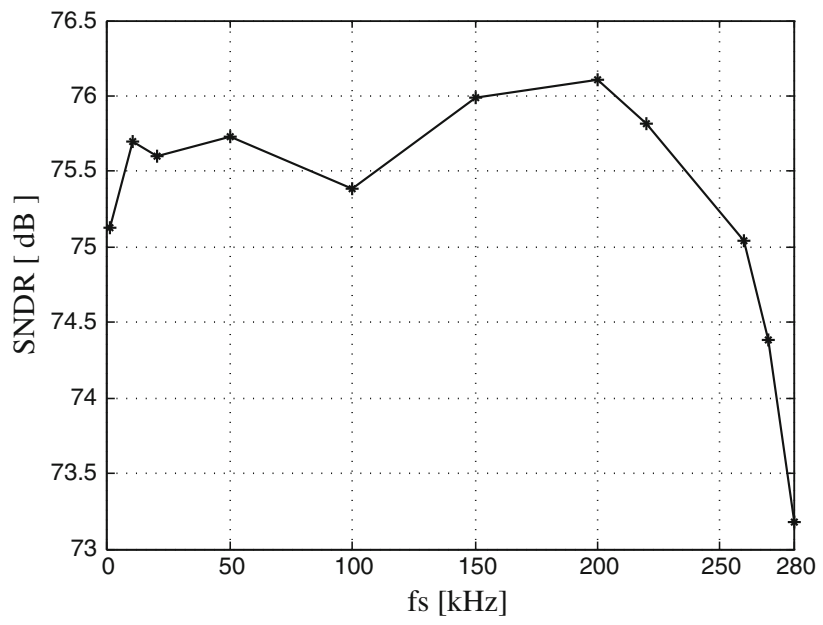

Fig. 14 Measured SNDR versus fs with near-DC input $\left(f_{\text {in }}=\frac{105}{4096} f_{s}\right)$ 

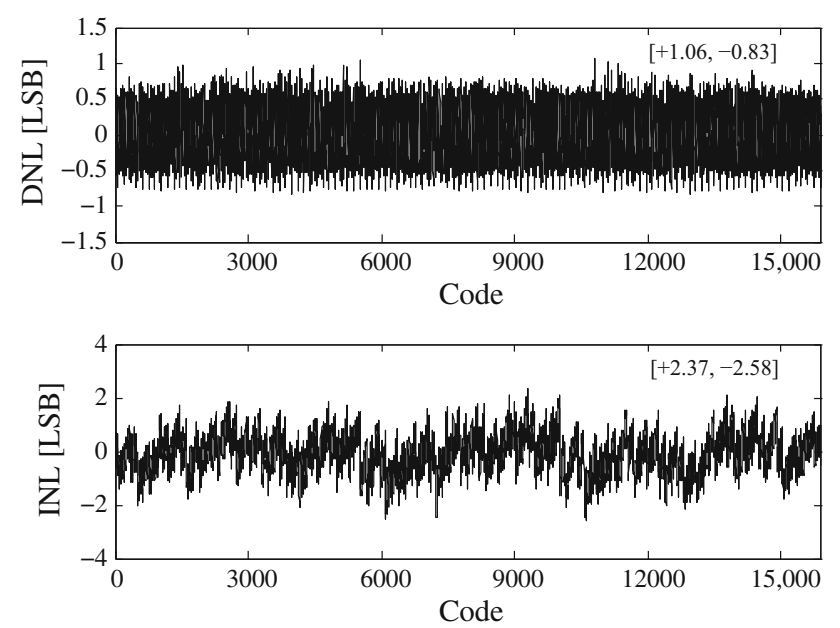

Fig. 15 Measured DNL and INL at $20 \mathrm{kS} / \mathrm{s}$
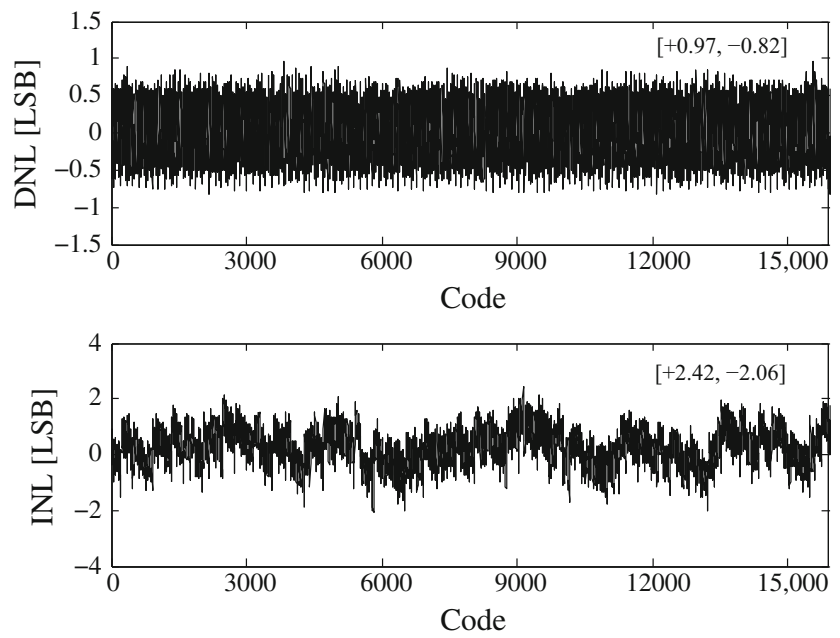

Fig. 16 Measured DNL and INL at $200 \mathrm{kS} / \mathrm{s}$
The peak DNL and INL are 1.06/-0.83 LSB and $2.37 /-2.58 \mathrm{LSB}$ for $20 \mathrm{kS} / \mathrm{s}$. For the $200 \mathrm{kS} / \mathrm{s}$ sampling rate, the peak DNL and INL are $0.97 /-0.82 \mathrm{LSB}$ and 2.42/-2.06 LSB, respectively.

With a supply voltage $3.3 \mathrm{~V}$ for analog and $3 \mathrm{~V}$ for the digital, the total power consumption of the ADC is $7.68 \mu \mathrm{W}$ at $f_{s}=20 \mathrm{kS} / \mathrm{s}$ and $96 \mu \mathrm{W}$ at $f_{s}=200 \mathrm{kS} / \mathrm{s}$. For $20 \mathrm{kS} / \mathrm{s}$ sampling rate, about $40.6 \%$ of total power was consumed by the digital, $26.9 \%$ by the CCP, $20 \%$ by the DAC and $12.5 \%$ by the comparator. For $200 \mathrm{kS} / \mathrm{s}$, the corresponding percentages are $32.7,41.8,15.5 \%$ and $10 \%$, respectively. Due to the increased $V_{\text {bias }}$, the CCP occupies more power with sampling rate of $200 \mathrm{kS} / \mathrm{s}$. Table 2 compares the performance of proposed ADC with other high-resolution pipelined SAR, pipelined and nyquist ADC. The Schreier FoM defined by

$\mathrm{FoM}=\mathrm{SNDR}+10 \log \left(\frac{\mathrm{BW}}{\mathrm{P}}\right)$

has been used to compare the performance of the ADCs. The proposed ADC achieves a peak FoM of $166.7 \mathrm{~dB}$ at 20 $\mathrm{kS} / \mathrm{s}$ and $166.3 \mathrm{~dB}$ at $200 \mathrm{kS} / \mathrm{s}$. Compared to the implementations in $0.35-\mu \mathrm{m}$ CMOS reported in $[20,21,22]$ this work achieves a best FoM. The achieved FoM is also comparable to the reported result from [8]. Hence, the proposed solution offers an attractive choice for designing the high resolution pipelined SAR ADC without designing a high DC gain OTA.

\section{Conclusion}

The three-stage capacitive charge pump as the gain-stage for a 14-bit two-stage pipelined SAR ADC was presented in this work. Due to the tunable bandwidth of CCP, The proposed ADC achieves a SNDR $>75 \mathrm{~dB}$ upto $260 \mathrm{kHz}$. Meanwhile the ADC also provides a competitive FoM among related works. By employing the $\mathrm{CCP}$ as the gain-

Table 2 Comparison of the ADC with other high-resolution ADCs

\begin{tabular}{|c|c|c|c|c|c|c|c|c|c|}
\hline & {$[20]$} & [21] & {$[22]$} & [8] & [9] & [11] & {$[23]$} & \multicolumn{2}{|c|}{ This work } \\
\hline CMOS (nm) & 350 & 350 & 350 & 130 & 65 & 28 & 65 & \multicolumn{2}{|l|}{350} \\
\hline Architecture & $\begin{array}{l}\text { Pipelined } \\
\text { with SC }\end{array}$ & $\begin{array}{r}\text { Pipelined } \\
\text { withSC }\end{array}$ & $\begin{array}{r}\text { Pipelined } \\
\text { withSC }\end{array}$ & $\begin{array}{l}\text { SAR- } \\
\text { assisted } \\
\text { withSC }\end{array}$ & $\begin{array}{l}\text { SAR-assisted } \\
\text { with Ring amplifier }\end{array}$ & $\begin{array}{l}\text { SAR-assisted } \\
\text { with DynamicSC }\end{array}$ & Nyquist SAR & \multicolumn{2}{|c|}{$\begin{array}{l}\text { SAR-assisted } \\
\text { with CCP }\end{array}$} \\
\hline Resolution (bit) & 14 & 14 & 12 & 14 & 13 & 14 & 14 & \multicolumn{2}{|l|}{14} \\
\hline Calibration & N0 & No & Yes & No & No & Yes & No & \multicolumn{2}{|l|}{ No } \\
\hline DC gain & $100 \mathrm{~dB}$ & - & $60 \mathrm{~dB}$ & $86 \mathrm{~dB}$ & $>80 \mathrm{~dB}$ & - & - & \multicolumn{2}{|l|}{$51.8 \mathrm{~dB}$} \\
\hline Area $\left(\mathrm{mm}^{2}\right)$ & 7.8 & - & 20.6 & 0.24 & 0.054 & 0.137 & 0.28 & \multicolumn{2}{|l|}{0.589} \\
\hline fs & $75 \mathrm{MS} / \mathrm{s}$ & $80 \mathrm{MS} / \mathrm{s}$ & $20 \mathrm{MS} / \mathrm{s}$ & $30 \mathrm{MS} / \mathrm{s}$ & $50 \mathrm{MS} / \mathrm{s}$ & $80 \mathrm{Ms} / \mathrm{s}$ & $10 \mathrm{kS} / \mathrm{s}$ & $20 \mathrm{kS} / \mathrm{s}$ & $200 \mathrm{kS} / \mathrm{s}$ \\
\hline SNDR (dB) & 74 & 74 & 72.5 & 70.8 & 71.5 & 68.0 & 78.1 & 75.6 & 76.1 \\
\hline SFDR (dB) & 94 & 100 & 84.4 & 87.8 & 87.0 & 80.7 & 88.5 & 90.9 & 92.7 \\
\hline Power & $318 \mathrm{~mW}$ & $1200 \mathrm{~mW}$ & $56.3 \mathrm{~mW}$ & $2.54 \mathrm{~mW}$ & $1.0 \mathrm{~mW}$ & $1.5 \mathrm{~mW}$ & $2.48 \mu \mathrm{W}$ & $7.68 \mu \mathrm{W}$ & $96 \mu \mathrm{W}$ \\
\hline FoM (dB) & 154.7 & 149.2 & 155 & 168.5 & 175.5 & 172.3 & 171.1 & 166.7 & 166.3 \\
\hline
\end{tabular}


stage, the high DC gain OTA in the SC integrator was avoided while also reducing the design complexity. So, we concluded that the multi-stage capacitive charge pump shows an another solution to achieve the function of the gain stage instead of using the SC integrator for the lowspeed, two-stage pipelined SAR ADC application.

Open Access This article is distributed under the terms of the Creative Commons Attribution 4.0 International License (http://crea tivecommons.org/licenses/by/4.0/), which permits unrestricted use, distribution, and reproduction in any medium, provided you give appropriate credit to the original author(s) and the source, provide a link to the Creative Commons license, and indicate if changes were made.

\section{References}

1. Harpe, P., Cantatore, E., \& van Roermund, A. (2014, February). An oversampled 12/14b SAR ADC with noise reduction and linearity enhancements achieving up to $79.1 \mathrm{~dB}$ SNDR. In ISSCC Dig. Tech. Papers, pp. 194-195.

2. Gunduz, D., Stamatiou, K., Michelusi, N., \& Zorzi, M. (2014). Designing intelligent energy harvesting communication systems. IEEE Communications Magazine, 52(1), 210-216.

3. Ho, C. K., \& Zhang, R. (2012). Optimal energy allocation for wireless communications with energy harvesting constraints. IEEE Transactions on Signal Processing, 60(9), 4808-4818.

4. Zhang, D., Bhide, A., \& Alvandpour, A. (2012). A 53-nW 9.1ENOB $1-\mathrm{kS} / \mathrm{s}$ SAR ADC in $0.13-\mu \mathrm{m}$ CMOS for medical implant devices. IEEE Journal of Solid-State Circuits, 47(7), $1585-1593$.

5. Harpe, P., Cantatore, E., \& Roermund, A. V. (2013, February). A 2.2/2.7 fJ/conversion-step 10/12b $40 \mathrm{kS} / \mathrm{s}$ SAR ADC with datadriven noise reduction. In 2013 IEEE International, solid-state circuits conference digest of technical papers (ISSCC), pp. 270-271.

6. Kapusta, R., Shen, J., Decker, S., Li, H., \& Ibaragi, E. (2013, February). A $14 \mathrm{~b} 80 \mathrm{MS} / \mathrm{s}$ SAR ADC with $73.6 \mathrm{~dB}$ SNDR in 65 nm CMOS. In ISSCC Dig. Tech. Papers, pp. 472-473.

7. Lee, C., \& Flynn, M. (2011). A SAR-assisted two-stage pipeline ADC. IEEE Journal of Solid-State Circuits, 46(4), 859-869.

8. Lee, H.-Y., Lee, B., \& Moon, U.-K. (2012, February). A 31.3 fJ/conversion-step $70.4 \mathrm{~dB}$ SNDR 30MS/s $1.2 \mathrm{~V}$ two-step pipelined ADC in $0.13 \mu \mathrm{m}$ CMOS. In ISSCC Dig. Tech. Papers, pp. 474-476.

9. Lim, Y., \& Flynn, M. (2015, February). A 1 mW 71.5 dB SNDR $50 \mathrm{MS} / \mathrm{s} 13 \mathrm{~b}$ fully differential ring-amplifier-based SAR-assisted pipeline ADC. In ISSCC Dig. Tech. Papers, pp. 1-3.

10. Verbruggen, B., Iriguchi, M., \& Craninckx, J. (2012, February). A $1.7 \mathrm{~mW} 11 \mathrm{~b} 250 \mathrm{MS} / \mathrm{s} 2 \times$ interleaved fully dynamic pipelined SAR ADC in $40 \mathrm{~nm}$ digital CMOS. In ISSCC Dig. Tech. Papers, pp. $466-468$.

11. van der Goes, F., Ward, C., Astgimath, S., Yan, H., Riley, J., Mulder, J., Wang, S., \& Bult, K. (2014, February). A 1.5 mW 68 dB SNDR $80 \mathrm{MS} / \mathrm{s} 2 \times$ interleaved SAR-assisted pipelined ADC in 28 nm CMOS. In ISSCC Dig. Tech. Papers, pp. 200-201.
12. Tripathi, V., \& Murmann, B. (2014, September). A $160 \mathrm{MS} / \mathrm{s}$, $11.1 \mathrm{~mW}$, single-channel pipelined SAR ADC with $68.3 \mathrm{~dB}$ SNDR. In 2014 IEEE proceedings of the custom integrated circuits conference (CICC), pp. 1-4.

13. Pylarinos, L. Charge pumps: An overview. http://uwire.google code.com/svn/trunk/doc/misc/chargepumps.

14. Chen, K., Harikumar, P., \& Alvandpour, A. (2016). Design of a $12.8 \mathrm{ENOB}, 1 \mathrm{kS} / \mathrm{s}$ pipelined SAR ADC in $0.35-\mu \mathrm{m}$ CMOS. Analog Integrated Circuits and Signal Processing, 86(1), 8798.

15. Baker, R. J. (2005). CMOS: Circuit design, layout, and simulation. New York: Wiley.

16. Saberi, M., \& Lotfi, R. (2014). Segmented architecture for successive approximation analog-to-digital converters. IEEE Transactions on VLSI System, 22(3), 593-606.

17. Wakimoto, T., Li, H., \& Murase, K. (2011). Statistical analysis on the effect of capacitance mismatch in a high-resolution successive-approximation ADC. IEEJ Transactions on Electrical and Electronic Engineering, 6(S1), S89-S93.

18. Sundstrom, T., Murmann, B., \& Svensson, C. (2009). Power dissipation bounds for high-speed Nyquist analog-to-digital converters. IEEE Transactions on Circuits and Systems I, 56(3), 509-518.

19. Abo, A., \& Gray, P. (1999). A 1.5-V, 10-bit, 14.3-MS/s CMOS pipeline analog-to-digital converter. IEEE Journal of Solid-State Circuits, 34(5), 599-606.

20. Yang, W., Kelly, D., Mehr, I., Sayuk, M., \& Singer, L. (2001). A 3-V 340-mW 14-b 75-Msample/s CMOS ADC with 85-dB SFDR at Nyquist input. IEEE Journal of Solid-State Circuits, 36(12), 1931-1936.

21. Bardsley, S., Dillon, C., Kummaraguntla, R., Lane, C., Ali, A., Rigsbee, B., et al. (2006). A 100-dB SFDR 80-MSPS 14-Bit 0.35$\mu \mathrm{m}$ BiCMOS Pipeline ADC. IEEE Journal of Solid-State Circuits, 41(9), 2144-2153.

22. Yuan, J., Fung, S. W., Chan, K. Y., \& Xu, R. (2012). A 12-bit 20 $\mathrm{MS} / \mathrm{s} 56.3 \mathrm{~mW}$ pipelined ADC with interpolation-based nonlinear calibration. IEEE Transactions on Circuits and Systems I, 59(3), $555-565$.

23. Zhang, D., \& Alvandpour, A. (2016). A 12.5-ENOB 10-kS/s redundant SAR ADC in 65-nm CMOS. IEEE Transactions on Circuits and Systems II: Express Briefs, 63(3), 244-248.

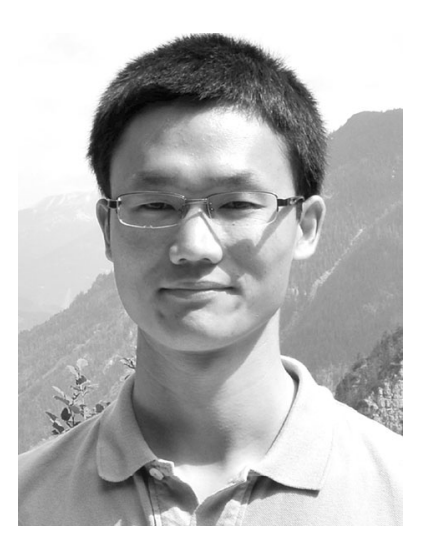

Kairang Chen received the B.Eng. degree in electronics and communication engineering from Guizhou University, China, in 2009, and the M.S. degree in communication engineering from Chongqing University, China, in 2012. Since 2012, he has been pursuing the Ph.D. degree in electrical engineering at Linköping University. His research interests include the design of lowpower and high-resolution analog-to-digital converters. 


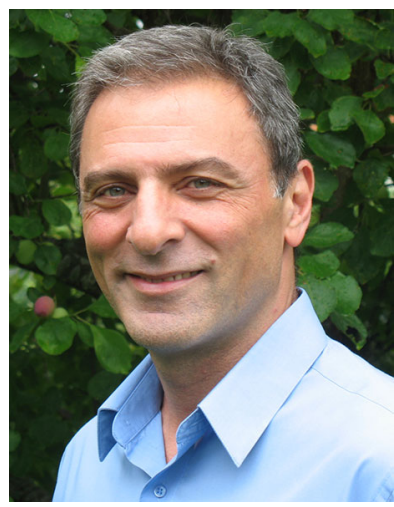

Atila Alvandpour received the M.S. and Ph.D. degrees from Linköping University, Sweden, in 1995 and 1999, respectively. From 1999 to 2003, he was a senior research scientist with Circuit Research Lab, Intel Corporation. In 2003, he joined the department of Electrical Engineering, Linköping University, as a Professor of VLSI design, and currently he is the head of the Integrated Circuits and Systems Division. His research interests include various issues in design of integrated circuits and systems in advanced nano-scale technologies, with special focus on data converters, sensor readout and data acquisition systems, energy-harvesting and power management systems, low-power wireless sensors, and high-performance digital/analog baseband and RF frontends for multi-Gigabit/s radio transceivers. He has published more than 100 papers in international journals and conferences, and holds 24 U.S. patents. Prof. Alvandpour is a senior member of IEEE, and has served as a member of technical program committees for many IEEE and other international conferences, including the IEEE Solid-State Circuits Conference, ISSCC, and the European Solid-State Circuits Conference, ESSCIRC. He has also served as guest editor for IEEE Journal of SolidState Circuits. 